\title{
Formative Research on the Heuristic Task Analysis Process
}

\author{
Ji-Yeon Lee
}

Charles M. Reigeluth

Corporate and educational settings
increasingly address more decision-making,
problem-solving and other complex cognitive
skills to handle complex cognitive, or heuristic,
tasks, but the ever-increasing need for
heuristic knowledge has outpaced the
refinement of task analysis methods for
heuristic expertise. Utilizing the Heuristic
Task Analysis (HTA) process, a method
developed for eliciting, analyzing, and
representing expertise in complex cognitive
tasks, a formative research study was
conducted on the task of group counseling to
further improve the HTA process. Implications
of the findings include the need for
incorporating various interview strategies and
techniques, developing strategies for working
with multiple experts, and considering the
level of task expertise of the analyst. A revised
version of the HTA process is presented based
on these implications.

Corporate and educational settings increasingly address more decision-making, problem-solving and other complex cognitive skills to handle complex cognitive, or heuristic, tasks, but the ever-increasing need for heuristic knowledge has outpaced the refinement of task analysis methods for heuristic expertise. Utilizing the Heuristic Task Analysis (HTA) process, a method developed for eliciting, analyzing, and representing expertise in complex cognitive tans, a formative research study was conducted on the task of group counseling to further improve the HTA process. Implications of the findings include the need for incorporating various interview strategies and techniques, developing strategies for working with multiple experts, and considering the level of task expertise of the analyst. A revised on these implications.
As our society in general and the workplace in particular become more complex, we are finding that a greater number of the activities that people undertake are relatively more complex cognitive tasks than ever before. Whether in $\mathrm{K}$ 12 education, higher education, corporate training, or any other context, to help people learn the heuristic (complex cognitive) elements of an expert's know-how, we must be able to identify those heuristics.

In relation to complex cognitive tasks, we find it helpful to think in terms of two major kinds of expertise-domain and task expertise. According to Reigeluth (1999), task expertise relates to the learner "becoming an expert in a specific task, such as managing a project, selling a product, or writing an annual plan" (p. 435), while domain expertise relates to the learner "becoming an expert in a body of subject matter not tied to any specific task, such as economics, electronics, or physics (but often relevant to many tasks)" (p. 435). Both procedural and declarative knowledge are important elements of both kinds of expertise. In this research, we focus on task expertise.

For task expertise, we find it helpful to think in terms of two major kinds of tasks: procedural and heuristic. Procedural tasks are "tasks for which experts use a set of steps, mental, physical, or both, to decide what to do when, such as a high school course on mathematics or a corporate training program on installing a piece of equipment for a customer." (Reigeluth, 1999, p. 435). Heuristic tasks are "tasks for which experts use causal models-interrelated sets of principles or guidelines-to decide what to do when, such as a high school course on thinking skills or 
a corporate training program on management skills" (Reigeluth, 1999, p. 435).

The distinction between procedural and heuristic tasks is similar to the distinction between well-structured and ill-structured domains (Fredericksen, 1984; Resnick, 1988; Simon, 1973). In reality most tasks are neither purely procedural nor purely heuristic, but some combination of the two. We have relatively powerful methodologies for analyzing the expertise that underlies procedural elements of a task (i.e., the mental and physical steps upon which an expert relies). But we do not have good methodologies for analyzing the expertise that underlies heuristic elements of a task. This situation is exacerbated by the reality that heuristic knowledge is frequently tacit rather than explicit-that is, experts are often unaware of the heuristics that guide their performance. Therefore, there is a strong need to develop task analysis methodologies for identifying heuristic knowledge.

This paper briefly reviews the current state of knowledge related to heuristic task analysis. Then it describes a formative research study that has been conducted to advance the development of one of those methodologies.

\section{CURRENT STATE OF KNOWLEDGE}

In the relevant literature, we identified knowledge elicitation, analysis, and representation as three important aspects of heuristic task analysis. Here we will examine various techniques that are frequently used in analysis, from traditional task analyses to heuristic task analyses, on the basis of guidance provided for those three aspects. We found that most techniques deal with procedural tasks rather than heuristic tasks, and those that deal with heuristic tasks focus primarily on how to elicit experts' knowledge. Furthermore, it is difficult to find research that provides tested guidelines on how to analyze and represent such knowledge.

\section{Knowledge Elicitation}

Knowledge elicitation refers to the extraction of domain-relevant knowledge directly from a human expert using various techniques Jones, Miles, \& Read, 1996). Research on knowledge elicitation has been conducted mostly in relation to expert systems that use expert knowledge to perform complex problem-solving and decisionmaking processes, and many studies reported that knowledge elicitation has continued to be the major bottleneck in the process of building knowledge-based systems (Cooke, 1994; Wood \& Ford, 1993).

Based on the belief that the better the data gathered in elicitation, the better the resulting model of expert knowledge, many researchers have been interested in identifying relevant knowledge elicitation techniques. Some of them have focused on the type of knowledge elicited by the technique, under the assumption that different elicitation methods tap different types of knowledge (Kitto \& Boose, 1987; Wielinga, Schreiber, \& Breuker, 1992). Others have categorized techniques according to the stage in the elicitation process in which the techniques are employed (Olson \& Biolsi, 1991; Shaw \& Woodward, 1989). There is also research that organizes the knowledge elicitation techniques based on the mechanics of the techniques themselves (Jones et al., 1996; Tomlinson \& Johnson, 1994).

One of the most comprehensive reviews of knowledge elicitation techniques has been done by Cooke (1994). She categorized various knowledge elicitation methods into three families on the basis of methodological similarity: (a) observations and interviews, (b) process tracing, and (c) conceptual techniques. Each family of techniques has its own advantages and disadvantages, and there seems to be no single family that is suited for every purpose. Indeed, the studies on various knowledge elicitation techniques have emphasized the notion that one should not rely on any single technique (Cooke, 1994; Hoffman, Crandall, \& Shadbolt, 1998). It seems that the major problem with knowledge elicitation techniques is not a shortage of methods but a lack of advice on how to select and apply those methods depending on the type of task and contextual constraints. In other words, there has been the general tendency to "name methods without providing much infor- 
mation on how to apply them" (Forsythe \& Buchanan, 1989, p. 435).

\section{Knowledge Analysis}

Recent studies have often used computer programs to automate the analysis process, representing a shift away from knowledge analysis in which human analysts are involved, toward more "nonhuman-based" approaches (Olson, Mechitov, \& Moshkovich, 1995; Sommer, Morik, Andre, \& Uszynski, 1994). For example, Benbenishty (1992) reported the use of a computer program for structured interviews in which the program constantly analyzed the expert's responses, finding inadequately covered areas, identifying apparent contradictions that should be clarified, and eliciting needed refinements (thus overlapping with the knowledge elicitation phase described earlier). He also described how sophisticated programs could be used in protocol analysis, partitioning transcribed protocols of the expert into segments on the basis of speech pauses. However, there is little guidance concerning the analysis of the data generated by interviews and observations, and without a systematic framework for analysis, the reliability and validity of such data have often been questioned by researchers (Cooke, 1994; Hoffman et al., 1998).

\section{Knowledge Representation}

Knowledge elicitation methods have mostly been used with a fairly narrow set of applications in mind, primarily the development of expert systems and artificial intelligence systems. Expert systems are computer systems designed to tackle problems normally thought to require the intervention of human experts, and the overall goal of knowledge elicitation is to externalize (or represent) knowledge in a form that can be implemented in such systems (Cooke, 1994; Hoffman et al., 1998; LaFrance, 1992).

Not surprisingly, a central problem for researchers has been to find ways to represent expert knowledge in a manner that computers can use. Some of the frequently used knowledge representation schemes include semantic networks, augmented transition networks, bites, frames, scripts, and grammar. They have been selectively used to construct systems that hierarchically organize information for tutoring, simulation, and parsing of natural language, depending on the type of problem being addressed (Nelson, 1989; Wenger, 1987).

It appears that different types of knowledge require different types of representation (Nelson, 1989; Wenger, 1987). For example, a method that-can best represent procedural knowledge might not be equally effective for heuristic knowledge. Knowledge representation is also often influenced by the purpose of its application. Building an expert system has been an important reason for conducting task analyses, and research on knowledge representation has been driven by that goal. As a consequence, there is a lack of research on knowledge representations to inform instructional designers on heuristic knowledge to use in instruction.

In the next section, we will introduce task analysis methodologies that are more focused on heuristic tasks.

\section{Cognitive Task Analysis (CTA)}

Having emerged as a new branch of applied psychology to better understand task domains and human experts who perform the tasks, cognitive task analysis (CTA) is a "broad area consisting of tools and techniques for describing the knowledge and strategies required for task performance" (Schraagen, Chipman, \& Shalin, 2000 , p. xiii). To elicit expert knowledge, researchers have tried various techniques that were first used by cognitive scientists studying human cognition in lab settings, including interview, focused discussion, protocol analysis, and simulation, as well as on-site observation (Black, Dimaraki, Esselstyn, \& Flanagan, 1995; Dehoney, 1995). Typically CTA involves either participants working on domain-free problems or knowledge workers who solve complex cognitive problems for a living (e.g., aviators or $\mathrm{X}$ ray technicians).

Dehoney (1995) suggested two phases in conducting CTA: (a) to derive a model of competen- 
cies for performing the task, and (b) to derive a performance model that specifies how practitioners actually perform the task. The goal of the first phase is to identify what people must be able to do to accomplish the task at a satisfactory level, what kinds of problems they must solve, what they must know, and how they must use this knowledge to solve problems. Based on the competency model, the second phase of analysis creates a performance model to improve people's performance.

In their recent, comprehensive efforts to review the types and conditions of various CTA methods and techniques in the literature and practice, Schraagen and his colleagues (2000) further elaborated the CTA process into four phases: (a) identifying and becoming generally familiar with the task; (b) identifying the nature of knowledge and determining the type of knowledge representation for later in the process; (c) implementing various knowledge elicitation techniques; and (d) packaging the elicited knowledge into CTA products. CTA incorporates the aforementioned three main components of heuristic task analysis (i.e., knowledge elicitation, knowledge analysis, and knowledge representation), and thus provides a coherent and logical framework for analyzing expert knowledge in complex cognitive skills.

However, the extent to which an expert's domain-specific skills and knowledge captured in one situation can be transferred to other domains (or different situations within the same domain) remains a controversial issue among researchers in evaluating the effectiveness of CTA. Furthermore, previous research studies on CTA tend not to have investigated the conditions under which various knowledge analysis, elicitation, and representation techniques are appropriate, and the implications of each of those techniques for instructional designers and trainers to design and deliver effective instruction on complex cognitive skills.

\section{The Heuristic Task Analysis Method}

Heuristic Task Analysis (HTA) is a method developed for eliciting, analyzing, and representing expertise in complex cognitive (or heuristic) tasks (Reigeluth, 1999). Traditional task analysis methods typically result in a list of tasks and subtasks stated in behavioral terms and framed in a procedural format (Gordon \& Gill, 1997; Ryder \& Redding, 1993). In contrast, the HTA process primarily focuses on analyzing relatively complicated, ill-structured tasks for which experts use such heuristic knowledge as principles, causal models, and guidelines (or rules of thumb) to decide what to do when, instead of being aware of, and deliberately following, a set of steps.

The HTA begins by asking the questions, What is the simplest version of the task that an expert has ever performed? What is the next simplest version? and so on (see Table 1). Identifying the boundary of the simplest yet still representative version of the task dramatically reduces the amount of knowledge and number of contextual factors that are necessary for expert performance. More important, starting with the simplest version allows analysts to study the phenomenon of expert performance under simpler, yet still real world, conditions and thus to do so in a more reliable fashion (Ericsson \& Charness, 1997).

The simplest version of the task is analyzed using a deductive approach that elicits, analyzes, and represents heuristic knowledge within each of its major kinds (guidelines, explanatory models, descriptive models, and metacognitive-decision rules) as well as any procedural knowledge (steps) that may be involved in the task. (For more information about the major kinds of heuristic knowledge, see Table 2.) This process is done using several cases that meet the conditions characterizing the simplest version of the task. It is also done with several experts, to address the problem of idiosyncrasy (see Table 1).

Once the simplest version is analyzed, then progressively more complex versions of the task are identified and analyzed in order to determine the additional knowledge that experts use to perform them. Table 1 summarizes the HTA process showing primarily procedural knowledge. For more detail that includes heuristics comprising HTA, see Appendix A.

The HTA process has not yet been rigorously tested and, therefore, is in need of further research. However, the most important research 
Phase I. Prepare for Analysis and Design

1. Lay the groundwork for your analysis and design.

1.1 Establish rapport with a task expert.

1.2 Explain the analysis process you will be using.

1.3 Ask the task expert about the nature of the task in general.

1.4 Identify the characteristics of the learners in general.

1.5 Identify the delivery constraints of the task in general.

Phase II. Identify the Simplest Version of the Task

2. Identify the simplest version. Help the task expert to identify the simplest version of the task that is fairly representative of the task as a whole, and to describe the conditions that distinguish that version from all other versions.

3. Analyze the organizing content. Analyze the organizing content (mostly heuristics and descriptive theories) for this version of the task.

3.1 Ask the task expert to think of one specific performance of the task to analyze, or to videotape a performance for you to review with the expert during the analysis.

3.2 Use a top-down approach to analyzing the content (the knowledge upon which the expert's performance is based). In other words, start by identifying the general categories of knowledge that an expert uses, then proceed to analyze each.

3.3 Ask the task expert to think of similar performances of the task that constitute a single version of the task. Use each such performance to broaden the steps, guidelines, explanatory models, descriptive models, and metacognitive-decision rules so that they represent the knowledge bases the task expert uses to deal with all performances for that version of the task.

3.4 If time and resources permit, find a second task expert with whom to repeat this entire process (Steps 1-3.3) to identify any alternative views of the task and the knowledge that underlies its performance. It may even be wise to repeat this process with several more task experts. And you may want to go back and ask each task expert what he or she thinks about the perspectives of the other task experts, in an effort to reconcile conflicts and select among alternative ways of thinking about and performing the task.

issue at this early stage of its development is not to prove the HTA process, but to improve it. Given the immaturity of our knowledge about how to analyze heuristic tasks, what is needed most at this time is developmental research-what Cronbach and Suppes (1969) referred to as "decision-oriented inquiry," as opposed to "conclusion-oriented inquiry." Therefore, our research question is:

\section{In what ways can the HTA process potentially be improved?}

To answer this question, it is also necessary to find out which parts of the method are working well and which parts are not working so well. Furthermore, to improve the HTA process, it will likely be important (a) to change parts of the method; (b) to provide more detailed guidance about how to accomplish particular parts of the method; and (c) to identify possible variations in the method for different situations, such as different kinds of heuristic tasks or even different kinds of task experts.
To answer this research question, we conducted a developmental research study designed to test the HTA process and generate guidance for further improvement. The study is described next, followed by results and some general conclusions.

\section{METHOD}

The formative research methodology (Reigeluth \& Frick, 1999) was used to explore how the HTA process can be improved when applied to the complex cognitive task of group counseling. Formative research is a kind of developmental research (Lijnse, 1995), action research, and design-based research or design experiment (Brown, 1992; Design-Based Research Collective, 2003) that is intended to improve design theory and instructional methods (Reigeluth \& Frick). In contrast to research on descriptive theory, which emphasizes validity.or how well the description matches the reality of "what is," research on design theory or method is more 
Table $2 \square$ Major kinds of heuristic knowledge

\begin{tabular}{|c|c|c|}
\hline $\begin{array}{l}\text { Kinds of } \\
\text { Knowledge }\end{array}$ & Definitions ${ }^{2}$ & Examples ${ }^{2}$ \\
\hline Guidelines & $\begin{array}{l}\text { Prescriptive principles or } \\
\text { "rules of thumb" that an } \\
\text { expert uses to attain the } \\
\text { goals for the specific } \\
\text { performance of the task }\end{array}$ & $\begin{array}{l}\text { - Participate in the ice breakers yourself with the rest of } \\
\text { the counseling group. } \\
\text { - Try to remember the participants' names and call them } \\
\text { by their first name as often as possible; it communicates } \\
\text { that you respect and care about them. } \\
\text { - Ask the participants to talk about at least one thing that } \\
\text { they normally would not tell others. }\end{array}$ \\
\hline Explanatory models & $\begin{array}{l}\text { A set of related reasons } \\
\text { that constitute a causal } \\
\text { model explaining why } \\
\text { the guidelines work }\end{array}$ & $\begin{array}{l}\text { - Compared to other types of ice breakers where people } \\
\text { take turns to introduce themselves, participants tend to } \\
\text { pay more attention to one another and be more active } \\
\text { speakers when paired to interview each other. } \\
\text { - When the participants know that they have to introduce } \\
\text { someone to the rest of the group, they tend to be more } \\
\text { willing to get to know that person. } \\
\text { By seeing the session leader take part in the group } \\
\text { exercise just like everyone else, participants tend to } \\
\text { accept the leader as part of the group and thus feel } \\
\text { more comfortable following his or her lead. }\end{array}$ \\
\hline Descriptive models & $\begin{array}{l}\text { A set of related causal } \\
\text { relationships characterizing } \\
\text { the phenomena or objects } \\
\text { with which the expert } \\
\text { works (as opposed to the } \\
\text { activities the expert performs }\end{array}$ & Interpersonal theories. \\
\hline $\begin{array}{l}\text { Metacognitive- } \\
\text { decision rules }\end{array}$ & $\begin{array}{l}\text { A set of rules the expert } \\
\text { uses to decide when to } \\
\text { use which steps, guidelines, } \\
\text { and descriptive models } \\
\text { during the specific } \\
\text { performance of the task } \\
\text { being analyzed }\end{array}$ & $\begin{array}{l}\text { - To decide which icebreaker activity to use is a } \\
\text { matter of preference. Because this step is instrumental, } \\
\text { the details of the activity do not matter as long as the } \\
\text { overall activity serves as an icebreaker at this early } \\
\text { stage of group formation. }\end{array}$ \\
\hline \multicolumn{3}{|c|}{$\begin{array}{l}\text { Notes: } \\
\text { 1. From Reigeluth (1999) } \\
\text { 2. From interviews of participating experts of group counseling in this study. For more details, see Appendix C. }\end{array}$} \\
\hline
\end{tabular}

concerned with preferability, the extent to which one method is better than other methods for achieving certain goals under certain circumstances. By creating or identifying an instance of a design theory and collecting formative data to improve that instance, one may develop a better understanding of how the design theory works in the field and thus be able to propose possible improvements for the theory-methods, which of course would need to undergo further testing.

Formative research can be used for improving an existing design theory or method or for developing a new grounded design theory or method, and in either case, it is a type of case study approach. According to Reigeluth and
Frick (1999), case studies can be classified as "designed" cases or "naturalistic" cases, depending on whether the situation under investigation is manipulated by the researcher or just observed.

This study used a designed case in which the HTA process was intentionally instantiated and manipulated by the researchers. It followed the steps suggested by Reigeluth and Frick (1999):

-1. Select a design theory or method: The method under investigation was the HTA process.

2. Design an instance of the theory: The designed instance in this study was the use of the HTA process to analyze group counseling, which is a predominantly heuristic task. 
3. Collect and analyze formative data on the instance: To identify problems and improve the HTA process, formative data were collected through interviews with task experts.

4. Revise the instance: This application of the HTA process was revised based on the data collected about how to improve it.

5. Repeat the data collection and revision cycle: The process of formative data collection and analysis was iterative. This study went through two rounds of data collection, analysis, and process revision.

6. Offer tentative revisions for the theory: The researcher formulated a set of tentative recommendations to improve the HTA process, based on the improvements made to the case.

The use of this six-step methodology is described in detail next.

Task

Group counseling was selected as the task to which to apply the HTA process. The nature of group counseling varies depending on the nature of the group that the leader facilitates, and this study focused on the counseling process for personal growth groups. The personal growth group is intended to help relatively healthy people to explore personal issues with which most people struggle at various transition periods in life and thus to function better on an interpersonal level (Stockton, Morran, \& Nitza, 2000).

Group counseling is predominantly a heuristic task with some procedural elements. It is procedural in that the activities of the group leader are largely determined by the stages that a group goes through (i.e., forming, norming, storming, and performing), and the leader cannot help the group to progress to the next stage without performing certain tasks at each stage (step). However, at a deeper level of analysis, the knowledge required for the leader to decide when and how to intervene is not a set of steps but a set of guidelines and principles, which is heuristic knowledge.

\section{Participants}

This study involved three participating task experts in group counseling to avoid idiosyncrasy. The first expert, a well-known authority in the field and the most experienced of the three task experts, was a university professor in the Counseling Department. The other two were advanced doctoral students in the same department, and they were selected based on the recommendations of the first expert. They were all experienced in conducting personal growth group counseling, and their expertise ranged from 3 to more than 20 years.

\section{Data Collection Methods}

Interviews. Semistructured, in-person interviews were used as the primary data collection method. There were two purposes of the interviews: (a) One was to use the HTA process for eliciting, analyzing, and representing the three experts' heuristic knowledge for performing the task of personal growth group counseling, and (b) the other was to find ways to improve the HTA process. Thus, the investigator played two roles during each interview: (a) one as a task analyst proficient in the HTA process and (b) the other as a researcher searching for ways to improve the HTA process. As task analyst, the investigator developed a set of interview questions (see Appendix B) based on the HTA process, but as researcher the investigator was not restricted to the HTA process or the related interview questions. Depending on the expert's response during each interview, the researcher noted revisions for the HTA process for the next interview. Furthermore, the investigator's approach as researcher to formatively evaluating the case (the implementation of the HTA process) was emergent. Thus, the overall interview process was flexible and reflective in nature.

Each expert was interviewed a minimum of two and maximum of four times, for about 30$90 \mathrm{~min}$ each time. For reasonable coverage of the expertise, given the purpose of the study, the number of interviews with each expert was adjusted during the course of the investigation (Merriam, 1998). In other words, the interviews 
were continued until a point of saturation or redundancy was reached.

The first interview followed the sequence of the HTA process described earlier (see Table 1 and Appendix A). It started with questions related to the nature of the task in general and then moved into questions related to identifying the simplest version of the task, asking the task expert to recall a specific instance (or case) that fit into the simplest version. For the second interview, several minor modifications were made based on the previous interview, and once the experts agreed on the boundaries of the simplest version of the task, the focus of the interviews moved into identifying specific decisions made and the underlying guidelines, explanatory models, descriptive models, and metacognitivedecision rules that the experts used.

Besides changes in the types of questions and the sequence of those questions, each interview used a slightly different technique from the previous ones, based on the formative data collected and analyzed. For example, after the fourth interview, the researcher (in the role of analyst) provided index cards (Davies, 1981) summarizing the critical incidents as a reference to help the experts recall specific heuristic knowledge already identified, whereas the first three interviews did not have any such reference materials.

Videotapes. Because of the confidential nature of group counseling, direct observation or videotaping of an expert's task performance (as called for by the HTA process) was not allowed. Instead, the researcher (as analyst) used a series of instructional videos that simulated group counseling sessions for beginning group leaders, to provide the analysts with a concrete case. ${ }^{1}$ The case fit the vast majority of the specifications the experts had identified for the simplest version of the task. The video series was developed by one of the participating experts, a renowned authority in the field of group counseling, and was one of the most broadly adopted instructional materials for clinical counseling courses.

1 The experts could add more details or delete certain parts, but mostly they accepted the scenario from the video without modification.
The series was composed of three videos, each of which covered different stages in the group counseling process. The study focused on the first three episodes, which dealt with the beginning stage of group counseling, and it used them as if they were recordings of the experts' task performance. ${ }^{2}$ Based on the video, each expert was asked to identify with one of the coleaders in the video series and explain the decisions of the coleaders based on his or her own group counseling experiences.

\section{Data Analysis and Interpretation Methods}

The HTA process is an iterative process: Finishing the first round of HTA is not the end of the study but the beginning of the second round of HTA; and the end of the second round is, again, the beginning of the third round; and so on. The investigator went through two rounds of HTA in this study.

Triangulation. To enhance the thoroughness of the data, this study involved three experts as data sources. Each of them played somewhat different roles during the interviews. During each round of data collection, the second expert provided the initial structure of the task setting and knowledge base. Then the first and third experts reviewed the knowledge elements, verified them, and provided additional information. There were a few times when the three experts did not agree with one another. In such cases, if situations could not be found to justify those differences, the first expert's judgment was accepted, as he was the most experienced group counselor.

Member checks. After each interview with an expert, the researcher transcribed the interview and took the summary and interpretations to the next interview for review. Through this process,

2 This was possible because all three experts who participated in this study were familiar with this video series. The first was the author of the video series. The other two had taken classes from the first and had studied the videos as class materials. 
the experts corrected errors or misconceptions by the researcher, and the researcher asked additional questions to clarify the information.

\section{DISCUSSION OF FINDINGS AND SUGGESTIONSFOR FURTHER IMPROVEMENT}

Overall, the initial HTA applied to this study yielded both procedural and heuristic knowledge that the participating experts used to guide their performance in group counseling. (For more detail, see Appendix C). The HTA interview protocols worked well in that the researcher (as analyst) could elicit steps and substeps involved in group counseling, and heuristic knowledge with which the experts performed each step. All three experts found their participation in the HTA interview process beneficial, because it provided opportunities to reflect on their own practice and become more aware of their underlying heuristic knowledge. At the same time, the researcher (as analyst) also faced challenges that were not initially addressed in the HTA. The following summarizes those issues based on the findings.

As mentioned earlier, there were two rounds of data collection in this study. The first round involved the initial interviews with the three experts. Instead of finishing with one expert and then starting with another, the researcher (as analyst) worked with the three experts simultaneously (but separately) because of their time schedules. This situation involved some tradeoffs. It worked well in the sense that the three experts could reach consensus on the simplest version of the task early in the HTA process. However, communicating with all three experts simultaneously was not easy for the analyst, because a difficult decision identified for discussion by one expert during the analysis was often difficult for another expert to pick up on to explain further the detailed decision-making process. Hence, the analyst had to spend most of the time during the initial interviews explaining to each expert the previous interviews with the other experts. Facing this problem, the researcher decided to use the existing instructional video series (with which all three experts were familiar, as described earlier in the Method sec- tion) as a frame of reference, instead of trying to build a new scenario based on each expert's differing experience.

The second problem the researcher encountered during the first round of HTA was that of lacking personal expertise in the task of group counseling. The researcher found that, to be able to push the expert to further elaborate his or her automatized (and hence subconscious) task expertise, the analyst needed to "speak the same language" as the expert and be able to prompt when the expert had difficulty in finding the right words. Without a certain level of expertise in the task domain, the researcher as task analyst had difficulty doing those jobs smoothly. The researcher tried to become familiar with the field by reading some introductory textbooks and articles written by the primary expert in this study. However, learning about the field was time consuming and could not be satisfactorily achieved within the time constraints.

The third problem was related to the difficulty of categorizing the types of knowledge underlying each decision made by the expert during the task analysis. Even though the researcher explained the differences among the various types of knowledge according to the HTA, such as guidelines and decision rules, often the experts had difficulties in distinguishing those concepts. Not being familiar with their field, the researcher had difficulty labeling the type of knowledge underlying certain decisions. The purpose of identifying the five types of knowledge specified by the HTA was to make sure that the experts did not overlook one of the important types of knowledge, but in this case the benefits of distinguishing among the types seemed not to be worth the extra time required.

The second round of HTA incorporated some new methods to deal with the problems found in the first round. First, the researcher summarized key incidents from the video series on index cards and used them as a reference during the interviews with the experts. This was very helpful in three ways: (a) it helped the experts to recall details about the task performance process; (b) it helped both analyst and experts to see the flow of the task performance process and get back on track when the experts got off task; 
and $(c)$ it saved a lot of time in revisiting previous points. One expert commented that the index cards forced him to be more precise during the review and revision process.

Second, the researcher as analyst used an inductive approach (identifying knowledge first, then categorizing it as to type) rather than the deductive approach (identifying knowledge within each type) suggested by the HTA process. In the first round, the experts had difficulties identifying the kind of knowledge underlying each decision, and spent a lot of time on that. Thus, in the second round the analyst asked the experts to describe all knowledge related to performing the task first, regardless of the type of knowledge, trying not to interrupt their cognitive process. Later the analyst categorized each piece of knowledge and then asked the experts to verify the categories. This inductive approach seemed to be more efficient than the deductive approach, especially for the less experienced experts.

Based on the findings of this study, the following changes are proposed as possible improvements:

- Incorporate various interview and observation techniques into the HTA process.

- Provide guidelines for working with multiple task experts having different levels of expertise.

- Provide different guidelines for analysts with different levels of task expertise.

- Use an inductive approach and later categorize the types of knowledge identified by experts during the analysis.

- Provide reference material during the interviews with task experts.

Each of these changes is described in detail below (see Table 3).

\section{Incorporate various interview and observation techniques}

In-person interview appeared to be an effective way to elicit the expert's covert heuristics and make them explicit by verbal explanation. However, the expert was not always able to verbalize his or her own thinking process. Thus, the analyst needed to develop her own sense of heuristics through observing the expert's task performance (in this case on video tape) or reviewing reference materials to fill in the gap between what was said and what remained unsaid. Especially because the analyst had little expertise in the task domain, direct and indirect observations helped her understand the workflow and task. Unfortunately, for a task that involves interaction with other people or requires confidentiality, such as group counseling in this study, direct observational techniques were not appropriate, and the analyst needed to find alternatives, such as simulated videotapes or staged cases. Given these considerations, specific suggestions for improving the HTA are hypothesized in the next paragraph.

\section{Develop strategies for working with multiple experts}

Using multiple experts was beneficial, but working with more than one expert required special strategies for the analyst. It is recommended that the analyst complete a series of interviews with one expert, then conduct the analysis with another, and so forth, so as to create a separate analysis for each expert rather than one single analysis. The advantages appear to be that, first, each expert would know what has transpired to date in the analysis, and second, similarities and differences in heuristic knowledge of the experts would be catalogued in separate analyses, making subsequent comparisons and discussions easy. In making this change, we also suggest that the analyst start working with the least experienced expert, and move on to the next least experienced expert. The less experienced experts' knowledge seemed to be less automatized, and thus those experts were more conscious of their own heuristic knowledge. They tended to provide more information than the more experienced experts. Later, the analyst should ask the more experienced experts to review the results from the previous interviews. The more experienced experts provided more insightful information, which made them better reviewers. Nevertheless, it worked well to have a focus-group interview with multiple experts in the beginning, to reach consensus on the simplest version of the task. 
Table $3 \square$ Strengths and weakness of the Heuristlc Task Analysis (HTA) process

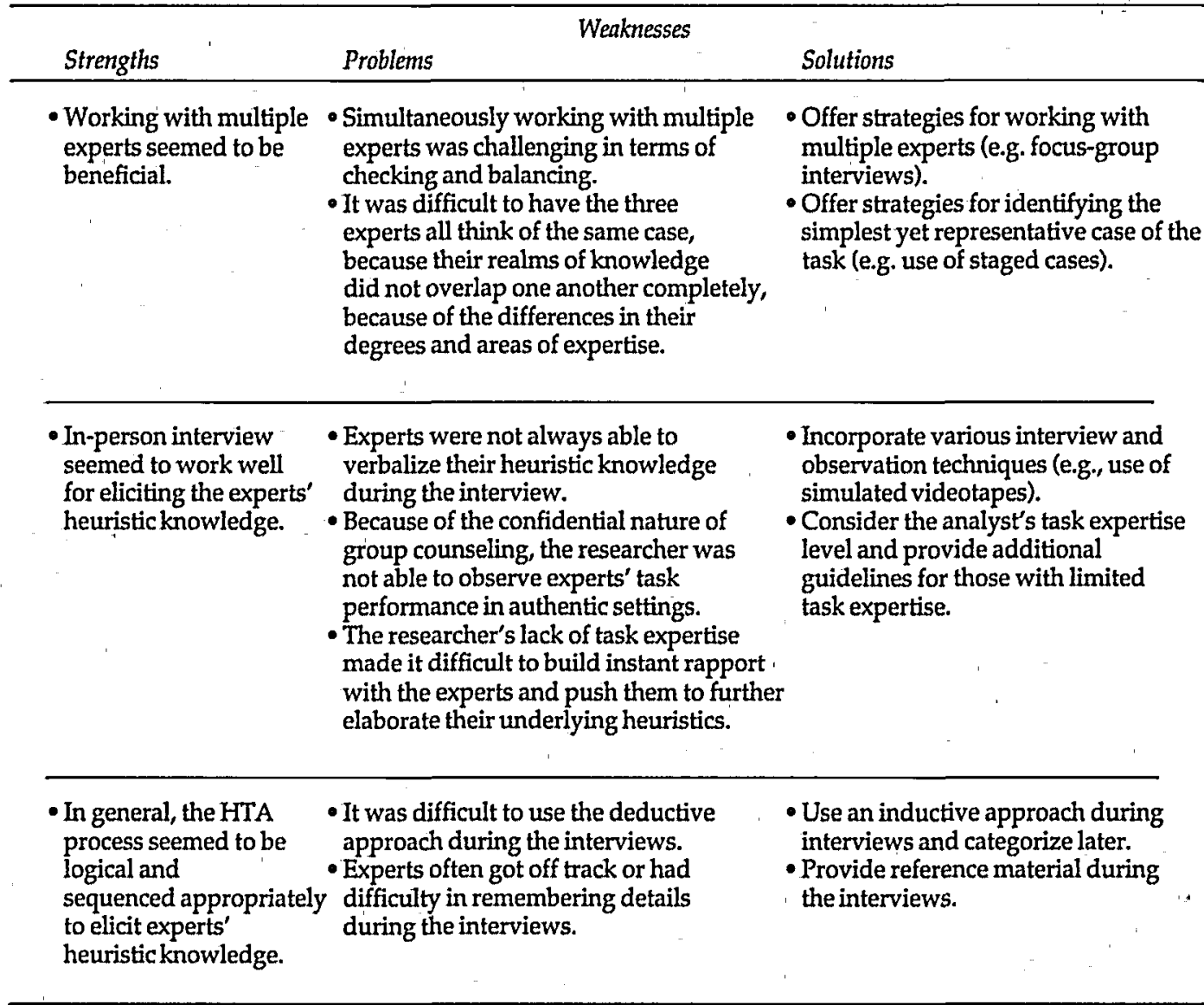

\section{Consider the analyst's task expertise level}

As Dehoney (1995) suggested, the analyst needs to learn enough about the task to be able to ask the right questions and understand the answers before being able to further explore the expert's heuristic knowledge. During interviews, the expert sometimes forgot to explain important things or was unable to verbalize them. Having a certain level of domain expertise would have helped the analyst to be aware of any missing knowledge from the expert's explanations. Besides that, the analyst's early lack of confidence in the task hindered building rapport with the experts throughout the analysis process. Thus, it is recommended that the HTA provide the following additional guidelines for analysts with limited task expertise:

- During preparation, review basic reference materials and become familiar with key con- cepts and terminology in the field. Such expertise could improve the analyst's rapport with the experts and the analyst's ability to help experts identify heuristic knowledge. Furthermore, it would be helpful for the analyst to observe the performance of the chosen case.

- It is likely that the more complicated the version of the task, the more task knowledge is required for the analyst. Thus, the analyst with limited task expertise should begin by, and focus on, identifying the simplest version of the task, rather than trying to expand the analysis to the next version.

Use an inductive approach, and later categorize the types of knowledge identified by experts during the analysis

Categorizing the experts' knowledge as steps, 
guidelines, explanatory models, descriptive models, and metacognitive-decision rules was difficult in this case, and its benefits appear not to have outweighed its costs. The benefits need to be clarified for the analyst. It worked better in this case to have the experts identify free-form whatever knowledge they could about the task, and later try to categorize it as to types (steps, guidelines, explanatory models, descriptive models, and metacognitive-decision rules) and fill in any gaps, rather than trying to get the experts to identify knowledge systematically within each type of knowledge.

\section{Provide reference materials during the interview}

When asked to describe the task performance process relying on memory, each expert tended to provide rather abstract explanations and forget to tell the details. Having access to some kind of reference material, such as index cards summarizing critical incidents, during the task performance or video-audio recordings of the expert's actual task performance, provided contextual information when needed and thus helped the expert to be more precise. During the iterative interview process, the visual aid also helped the expert to keep on track. Especially under circumstances with time constraints, using index cards appears to be a way to save time.

\section{CONCLUSION}

The initial HTA worked relatively well in eliciting, analyzing, and representing heuristic knowledge underlying the experts' performance in group counseling. Based on the formative data in this research study, a tentative revision of the HTA process is presented in Appendix A, in comparison to the original process. Formative research data indicate that the revised HTA process would have been even more effective for the investigated case.

As with most design-based, formative research studies (Design-Based Research Collective, 2003), the focus of this study was not to confirm the HTA process but to improve it by providing a rich description of a case in which the HTA process was applied to a task domain. It should be noted that such descriptions could enhance our understanding of how, when, and why the process works in that particular setting, yet not guarantee the universal applicability across domains. Although the above suggested revisions are based on the actual findings of this study, their applicability beyond the case of group counseling has not yet been empirically tested.

Therefore, it remains to be seen whether or not this revised process will also work well for other cases of analyzing heuristic tasks. The data in this study suggest that additional guidance might be beneficial for conducting a heuristic task analysis. It is our hope that this study will encourage others to conduct additional research to improve the available guidance for analyzing heuristic tasks.

Ji-Yeon Lee [leejy@gwm.sc.edu] is Assistant Professor of Educational Technology in the Department of Educational Psychology at the University of South Carolina.

Charles M. Reigeluth is Professor of Instructional Systems Technology in the School of Education at Indiana University.

\section{REFERENCES}

Benbenishty, R. (1992). An overview of methods to elicit and model expert clinical judgment and decision making. Socinl Science Review, 66(4), 598616.

Black, J.B., Dimaraki, E., Esselstyn, D., \& Flanagan, R.(1995). Using a knowledge representations approach to cognitive task analysis. Proceedings of the 1995 Annual National Convention of the Association for Educational Communications and Technology (AECT).

Brown, A.L. (1992). Design experiments: Theoretical and methodological challenges in creating complex interventions in classroom settings. Journal of the Learning Sciences, 2, 141-178.

Cooke, N.J. (1994). Varieties of knowledge elicitation techniques. International Joumal of Human-Computer Studies, 41(6), 801-849.

Cronbach, L.J., \& Suppes, P. (Eds.). (1969). Research for tomorrow's schools: Disciplined inquiry for education. New York: Macmillan.

Davies, I.K. (1981). Task analysis for reliable human performance. Performance and Instruction, 20(2), 4-7.

Dehoney, J. (1995). Cognitive task analysis: Implications for the theory and practice of instructional design. Paper 
presented at the Association for Educational Communications and Technology, Anaheim, CA.

Design-Based Research Collective. (2003). Designbased research: An emerging paradigm for educational inquiry. Educational Researcher, 32(1), 5-8.

Ericsson, K.A., \& Charness, N. (1997). Cognitive and developmental factors in expert performance. In P.J. Feltovich, K.M. Ford, \& R.R. Hoffman (Eds.), Expertise in context: Human and machine (pp. 3-41). Menlo Park, CA: American Association for Artificial Intelligence.

Forsythe, D.E., \& Buchanan, B.G. (1989). Knowledge acquisition for expert systems: Some pitfalls and suggestions. IEEE Transactions on Systems, Man, and Cybernetics, 19, 435-442.

Fredericksen, N. (1984). Implications of cognitive theory for instruction in problem solving. Review of Educational Research, 7, 38-40.

Gordon, S.E., \& Gill, R.T. (1997). Cognitive task analysis. In C.E. Zsambok \& G. Klein (Eds.)., Naturalistic decision making (pp. 131-140). Mahwah, NJ: Lawrence Erlbaum Assoc.

Hoffman, R.R., Crandall, B., \& Shadbolt, N. (1998). Use of the critical decision method to elicit expert knowledge: A case study in the methodology of cognitive task analysis. Human Factors, 40(2), 254-276.

Jones, S.R., Miles, J.C., \& Read, M.W. (1996). A comparison of knowledge elicitation methods. Expert Systems, 13(4), 277-295.

Kitto, C.M., \& Boose,J.H. (1987). Choosing knowledgeacquisition strategies for knowledge acquisition tasks. Paper presented at the WESTEX-87 Western Conference on Expert Systems.

LaFrance, M. (1992). Excavation, capture, collection and creation-Computer scientists' metaphors for eliciting human expertise. Metaphor and Symbolic Activity, 7(3-4), 135-156.

Lijnse, P.L. (1995). "Developmental research" as a way to an empirically based "didactical structure" of science. Science Education, 79(2), 189-199.

Merriam, S.B. (1998). Qualitative research and case study applications in education. San Francisco: Jossey-Bass, Inc.

Nelson, W.A. (1989). Artificial intelligence knowledge acquisition techniques for instructional development. Educational Technology Research and Development, 37(3), 81-94.

Olson, D.L., Mechitov, A.I., \& Moshkovich, H.M. (1995). The role of rules and examples in the process of knowledge acquisition in direct classification tasks. Expert Systems with Applications, 8(1), 203-212.

Olson, J.R., \& Biolsi, K.J. (1991). Techniques for repre- senting expert knowledge. In K.A. Ericsson \& J. Smith (Eds.), Toward a general theory of expertise (pp. 240-285). Cambridge: Cambridge University Press.

Reigeluth, C.M. (1999). The elaboration theory: Guidance for scope and sequence decisions. In C.M. Reigeluth (Ed.), Instructional-design theories and models: A new paradigm of instructional theory. Mahwah, NJ: Lawrence Erlbaum Associates.

Reigeluth, C.M., \& Frick, T.W. (1999). Formative research: A methodology for creating and improving design theories. In C.M. Reigeluth (Ed.), Instructional-design theories and models: $A$ new paradigm of instructional theory (Vol. 2) (pp. 633-651), NJ: Lawrence Erlbaum Associates.

Resnick, L. (1988). Treating mathematics as an ill-structured discipline. In R.I. Charles \& E.A. Silver (Eds.), The teaching and assessing of mathematical problem solving (pp. 32-60). Hillsdale, NJ: Lawrence Erlbaum Associates.

Ryder, J.M., \& Redding, R.E. (1993). Integrating cognitive task analysis into instructional systems development. Educational Technology Research and Development, 41(2), 75-96.

Schraagen, J.M., Chipman, S.F., \& Shalin, V.L. (Eds.). (2000). Cognitive task analysis. Mahwah, NJ: Lawrence Erlbaum Associates, Publishers.

Shaw, M.L.G., \& Woodward, J.B. (1989). Mental models in the knowledge acquisition process. Paper presented at the AAAI, Alberta, Canada.

Simon, H.A. (1973). The structure of ill structured problems. Artificial Intelligence, 23, 181-201.

Sommer, E., Morik, K., Andre, J.M., \& Uszynski, M. (1994). What on-line machine learning can do for knowledge acquisition-A case study. Knowledge Acquisition, 6(4), 435-460.

Stockton, R., Morran, D.K., \& Nitza, A.G. (2000). Processing group events: A conceptual map for leaders. Journal for Specialists in Group Work, 25 (4), 343-355.

Tomlinson, C.M., \& Johnson, L. (1994). Notes on techniques developed for knowledge elicitation. Systems Research and Information Science, 6(4), 179-185.

Wenger, E. (1987). Artificial intelligence and tutoring systems. Los Altos, CA: Morgan Kaufmann.

Wielinga, B.J., Schreiber, A.T.H., \& Breuker, J.A. (1992). KADS: A modeling to knowledge engineering. Knowledge Acquisition, 4, 5-53.

Wood, L.E., \& Ford, J.M. (1993). Structuring interviews with experts during knowledge elicitation. International Journal of Intelligent Systems, 8(1), 71-90. 
Appendix A $\square$ A comparison of the original and revised Heuristic Task Analysis (HTA) process

Original HTA Process Revised HTA Process

Phase I. Prepare for Analysis and Design

1. Prepare. Lay the groundwork for your analysis and design.

1.1 Establish rapport with a task expert.

1.2 Explain the analysis process you will be using.

1.3 Ask the task expert about the nature of the task in general.

1.4 Identify the characteristics of the learners in general.

1.5 Identify the delivery constraints of the task in general.
[Note: Changes and additions are in italics.]

1. Decide on a task to analyze and be clear about the reasons for analyzing it.

2. Make sure you have enough task knowledge to have a good command of terminology and key ideas.

- If you don't have enough task knowledge, review basic reference materials and try to become familiar with key concepts and jargon in the field.

3. Make sure you have enough knowledge about the uses of the task description.

- If the task description will be used primarily for deciding on the content and sequence of instruction, identify the characteristics of the learners in general and identify the delivery constraints of the instruction in general.

4. Arrange to interview multiple experts.

- Identify at least 2 or 3 experts to interview.

- Plan to complete the analysis with one expert before initiating the analysis with another.

- Plan to interview the least experienced expert first and proceed to interview progressively more experienced experts in order. The less experienced experts are likely to have less automatized knowledge, and thus be more conscious of their own heuristic knowledge. They tend to provide more information than the more experienced experts. On the other hand, the more experienced experts can provide more insightful information, which makes them better reviewers.

- Ask one or more of the task experts to record their performance of a very simple version of the task, and review the recorded material in advance of the analysis; or observe the task expert's task performance (more than once if possible).

5. Prepare in conjunction with the first (next) task expert.

- Establish rapport with the task expert.

- Introduce the HTA process to the expert.

- Explain basic terms (i.e. guidelines, explanatory models, etc.).

6. Prepare for the interview.

- Prepare interview materials (e.g., index cards to summarize critical incidents during task performance).

- Practice the HTA interview process if you are not very experienced in it.

- Arrange the interview logistics (e.g., reserve a conference room where you can work without interruptions).
Phase II. Identify the First Learning Episode

2. Identify the simplest version. Help the task expert identify the simplest version of the task that is fairly representative of the task as a whole, and to describe the conditions that distinguish that version from all other versions.

- You may want to use some other criteria in addition to simple and representative, such as common (how frequently performed the version
7. Identify the simplest version. Hold a focus group interview with multiple task experts, and help them reach consensus on the simplest version of the task that is fairly representative of the task as a whole. Also help them describe the conditions that distinguish that version from all other versions.

- You may want to use some other criteria in addition to simple and representative, such as common (how frequently performed the version of the task is) and safe (how much risk there is to the learner, other people, and/or the equipment).

- It may be helpful to have the expert briefly discuss closely related 
of the task is) and safe (how much risk there is to the learner and/or the equipment).

- Ask the task expert to recall the simplest case she or he has ever seen. The simplest version will be a class of similar cases. Then check to see how representative it is of the task as a whole.

- It may be helpful to start by identifying some of the major versions of the task and the conditions that distinguish when one version is appropriate versus another.

- Thinking of different conditions helps to identify versions, and thinking of different versions helps to identify conditions. Hence, it is wise to do both simultaneously (or alternately).

- There is no single right version to choose as the "simplest." It is usually a matter of trade-offs. The very simplest version of the task is usually not very representative of the task as a whole. The more representative the simple version can be, the better, for it provides a more useful schema to which learners can relate subsequent versions.

- It may be wise to go through this process with several task experts before going on to Step 3. You may find it necessary to take steps to resolve differences of opinion about which is the best "simplest version" to use.

3. Analyze the organizing content. Analyze the organizing content (mostly heuristics and descriptive theories) for this version of the task.

3.1 Ask the task expert to think of one specific performance of the task to analyze, or to videotape a performance for you to review with the expert during the analysis.

3.2 Use a top-down approach to analyzing the content (the knowledge upon which the expert's performance is based). In other words, start by identifying the general categories of knowledge that an expert uses, then proceed to analyze each.

- Ask the task expert:

a. to describe each decision that the task expert made

b. to identify the kinds of knowledge that the task expert drew upon to make the decision

c. to describe the specific knowledge tasks and clearly distinguish between the main task and the related tasks during the remainder of the analysis.

- Ask the task experts to recall the simplest case they have ever seen. The simplest version will be a class of similar cases. Then check to see how representative it is of the task as a whole.

- It may be helpful to start by identifying some of the major versions of the task and the conditions that distinguish when one version is appropriate versus another.

- Thinking of different conditions helps to identify versions, and thinking of different versions helps identify conditions. Hence, it is wise to do both simultaneously (or alternately).

- There is no single right version to choose as the "simplest." It is usually a matter of trade-offs. The very simplest version of the task is usually not very representative of the task as , a whole. The more representative the simple version can be, the better, for it provides a more useful schema to which learners can relate subsequent versions.

- It is wise to go through this process with several task experts together and reach consensus before going on to Step 8. You may find it necessary to take steps to resolve differences of opinion about which is the best "simplest version" to use.

8. Analyze the organizing content. With the least experienced expert you have not yet interviewed, analyze the organizing content (mostly heuristics and descriptive theories) for this - version of the task.

8.1 Review the recorded material (or any other visual aid) with the task expert.

8.2 Ask the task expert to think of and describe one specific performance of the selected version of the task to focus on for your analysis, or ask if a videotaped performance would be a good case for you to focus on with the expert during the analysis.

- It is often helpful to have a videotape of a typical performance of the simplest version of the task, so you and the task expert can review it during the analysis process, but asking the task expert to recall one specific performance and keep it in mind throughout the process is a more convenient and inexpensive, albeit often less effective, alternative.

- If you don't have a videotape, It may be helpful to have the expert describe contextual information and particulars of the specific performance, describing how the expert began the case, how it progressed (in sequence), how participants reacted, and how the expert dealt with any problems that arose.

- It may be helpful to prioritize the problems or concerns that arose and the decisions or actions that the expert used to deal with them.

8.3 Decide whether to use a deductive or inductive approach to analyzing the content (the knowledge upon which the expert's performance is based). If deductive, use Step 8.4 and skip Step 8.5. If inductive, skip Step 8.4 and use Step 8.5.

8.4. If deductive approach, start by explaining the general categories of knowledge that an expert uses, then proceed to analyze each.

- Ask the task expert:

a. to describe each decision that the task expert made, 
within each kind of knowledge that the task expert used

- The kinds of knowledge are likely to include:

a. steps (procedural knowledge)

b. guidelines or rules of thumb (heuristic knowledge)

c. explanatory models (which explain why the guidelines work)

d. descriptive models (which describe the phenomena with which the task expert interacts)

e. metacognitive-decision rules (which the task expert uses to decide which steps, guidelines, and descriptive knowledge, to use when).

- It is generally helpful to start by asking the task expert if there are any steps or phases of activities that are always performed for this version of the task. If so, perform a procedural task analysis to identify the sequence of steps and to see if any of those steps can be broken down into substeps, but those substeps must be ones that an expert thinks of and uses routinely in performing that version of the task.

- For guidelines, use the following process: a. Identify the goals for this specific performance of the task under its conditions.

b. Identify all the important considerations for attaining each goal. Considerations are the major categories of causal factors that influence performance of the task. If there are a lot of causal factors for a consideration, it is useful to identify subconsiderations for it.

c. Identify all the important causal factors for each consideration (or. subconsideration).

d. Analyze each causal factor to identify all guidelines prescriptive principles or "rules of thumb") that an expert uses to account for this consideration.

- For explanatory models, use the following process:

a. For each guideline, ask the task expert for the reasons why she or he believes it works.

b. For interrelated guidelines, you are likely to identify a set of related reasons that constitute a causal model or models. Be sure to look for multiple causes for each effect and multiple effects for each cause. Also look for chains of causes and effects, and b. to identify the kinds of knowledge that the task expert drew upon to make the decision, and

c. to describe the specific knowledge within each kind of knowledge that the task expert used.

- The kinds of knowledge are likely to include:

a. steps (procedural knowledge),

b. guidelines or rules of thumb (heuristic knowledge),

c. explanatory models (which explain why the guidelines work),

d. descriptive models (which describe the phenomena with which the task expert interacts), and

e. metacognitive-decision rules (which the task expert uses to decide which steps, guidelines, and descriptive knowledge, to use when).

- It is generally helpful to start by asking the task expert if there are any steps or phases of activities that are always performed for this version of the task. If so, perform a procedural task analysis to identify the sequence of steps and to see if any of those steps can be broken down into substeps, but those substeps must be ones that an expert thinks of and uses routinely in performing that version of the task.

- For guidelines, use the following process:

a. Identify the goals for this specific performance of the task under its conditions. It may help to have the expert explain the goals in task-specific terms rather than in abstract terms and to think of the goals as ideal outcomes.

b. Identify all the important considerations for attaining each goal. Considerations are the major categories of causal factors that influence performance of the task. If there are a lot of causal factors for a consideration, it is useful to identify subconsiderations for it.

c. Identify all the important causal factors that relate to each consideration (or subconsideration).

d. Analyze each causal factor to identify all guidelines (prescriptive principles or "rules of thumb") that an expert uses to account for this consideration.

- For explanatory models, use the following guidelines:

a. For each guideline, ask the task expert for the reasons why she or he believes it works.

b. For interrelated guidelines, you are likely to identify a set of related reasons that constitute a causal model or models. Be sure to look for multiple causes for each effect and multiple effects for each cause. Also look for chains of causes and effects, and explore probabilities for each causal factor to have each effect.

- For descriptive models, use the following guidelines:

a. Ask the task expert what phenomena influenced this particular performance of the task.

b. Try to identify all causal relationships that characterized those phenomena.

c. Be sure to look for multiple causes for each effect and multiple effects for each cause. Also look for chains of causes and effects, and explore probabilities for each causal factor to have each effect.

- For metacognitive-decision rules, find out what rules the task expert used to decide when to use which steps, guidelines, and descriptive models during the specific 
explore probabilities for each causal factor to have each effect.

- For descriptive models, use the following process:

a. Ask the task expert what phenomena influenced this particular performance of the task.

b. Try to identify all causal relationships that characterized those phenomena.

c. Be sure to look for multiple causes for each effect and multiple effects for each cause.

d. Look for chains of causes and effects, and explore probabilities for each causal factor to have each effect.

- For metacognitive/decision rules, find out what rules the task expert used to decide when to use which steps, guidelines, and descriptive models during the specific performance of the task being analyzed.

a. It is wise to query the task expert about any of these kinds of knowledge that are not initially described to you for each decision the task expert made in this specific performance of the task.

3.3 Ask the task expert to think of similar performances of the task that constitute a single version of the task. Use each such performance to broaden the steps, guidelines, explanatory models, descriptive models, and metacognitive-decision rules so that they represent the knowledge bases the task expert uses to deal with all performances for that version of the task.

3.4 If time and resources permit, find a second task expert with whom to repeat this entire process (Steps 1-3.3) to identify any alternative views of the task and the knowledge that underlies its performance.

3.5 It may even be wise to repeat this process with several more task experts. And you may want to go back and ask each task performance of the task being analyzed.

a. It is wise to query the task expert about any of these kinds of knowledge that are not initially described to you for each decision the task expert made in this specific performance of the task.

b. If the expert uses words such as know, feel, see, understand, like, determine, and decide, that may be an indication that heuristic knowledge underlies that particular performance.

c. It is often helpful to periodically ask the expert some questions about the chosen case, to keep the analysis focused on the flow of that version of the task.

$d$. It is useful to help the expert think about ways the specific case fell short of how it should have been done (an ideal case for this version of the task) and to have the expert offer guidelines for how this specific case should have been done.

e. It is wise to have some kind of reference material (e.g., index cards summarizing critical incidents of the task or videotapes of the task) to provide contextual information and cues and to help the expert be more precise. During the iterative interview process, the visual aid also helps the expert keep on track. Especially under circumstances with time constraints, using index cards is one of the ways to save time.

f. It may be helpfil to use index cards for all of these kinds of knowledge, filling them out with the task expert during the analysis process with one piece of knowledge per card, and arrange the cards in some order on a table in front of both of you, so you can easily switch from one part or aspect of the task to another.

8.5 If inductive approach, ask the expert to describe each decision and the process through which she or he went to make each decision.

- After the interview, try to categorize each piece of heuristic knowledge according to these categories:

a. steps (procedural knowledge),

b. guidelines or rules of thumb (heuristic knowledge),

c. explanatory models (which explain why the guidelines work),

d. descriptive models (which describe the phenomena with which the task expert interacts), and

e. metacognitive-decision rules (which the task expert uses to decide which steps, guidelines, and descriptive knowledge to use when).

- Be sure to "member check" the interview results with the expert in a later interview to verify or identify the types of knowledge underlying each decision.

8.6 Ask the task expert to think of similar performances of the task that are within the realm of the version of the task you are currently analyzing. Use each such performance to broaden the steps, guidelines, explanatory models, descriptive models, and metacognitive-decision rules so that they represent the knowledge bases the task expert uses to deal with all performances for that version of the task.

8.7 Repeat this entire process (Steps 5-8.6) with the next least experienced task expert to identify any alternative views of the task and the knowledge that underlies its performance. 


\section{Appendix A $\square$ Continued}

Original HTA Process

expert what she or he thinks about the perspectives of the other task experts, in an effort to reconcile conflicts and select among alternative ways of thinking about and performing the task.

\section{Revised HTA Process}

- For each more experienced expert, you should summarize the previous description of the task and ask the expert to review it, in an effort to reconcile conficts and select among alternative ways of thinking about and performing the task. The more experienced experts can provide more insightful information, which makes them better reviewers.

\section{Appendix B $\square$ Intervlew questions}

Questions related to the nature of the task in general:

1. How do you perceive group counseling? Is it procedural, heuristic, or both?

2. If it's procedural, in what circumstances?

3. If it's heuristic, in what circumstances?

4. If it's both, in what circumstances?

Questions related to the simplest version of the task:

5. Could you think of any group counseling session that is the simplest but still representative?

- Tell me about the setting:

-When did you lead the session?

- How many participants did you have?

-What kinds of people were they?

-What was the goal of the session?

-What are the main considerations to achieve the goal?

- Tell me about major decisions you made during the session:

-What was the procedure you followed (if there was any)?

-What were the major decision-points at the session? (i.e., When did you intervene and how did you intervene?)

- Tell me what led you to make such decisions:

-What are the decision-rules at each decision-point?

-What are the alternatives at each decision-point?

- How do the decision rules work?

-Could you explain why each of the rules worked or did not work?

-What would you do differently if you encountered similar cases in the future?

\section{Appendix C $\square$ Heuristic Task Analysis (HTA) of group counseling process: A summary}

\footnotetext{
Goals and Conditions

Goals and Sub-goals:

1. Search for the structure for the group members to disclose themselves.

2. Search for the structure for the group members to provide feedback to each other.

Conditions or Variables to be considered:

1. Number of members in group

2. Personalities of group members

3. Patterns of group dynamic

4. Time constraints

5. Group characteristics (i.e. group composition of males and females)
}

[Note: Superscript numbers in the left column correspond to the heuristics in the right column]

1.1. Provide an environment for the group members to feel safe to express their problems.

2.1. Provide an environment for the group members to feel safe to provide feedback to each other. 
Appendix $\subset \square$ (continued)

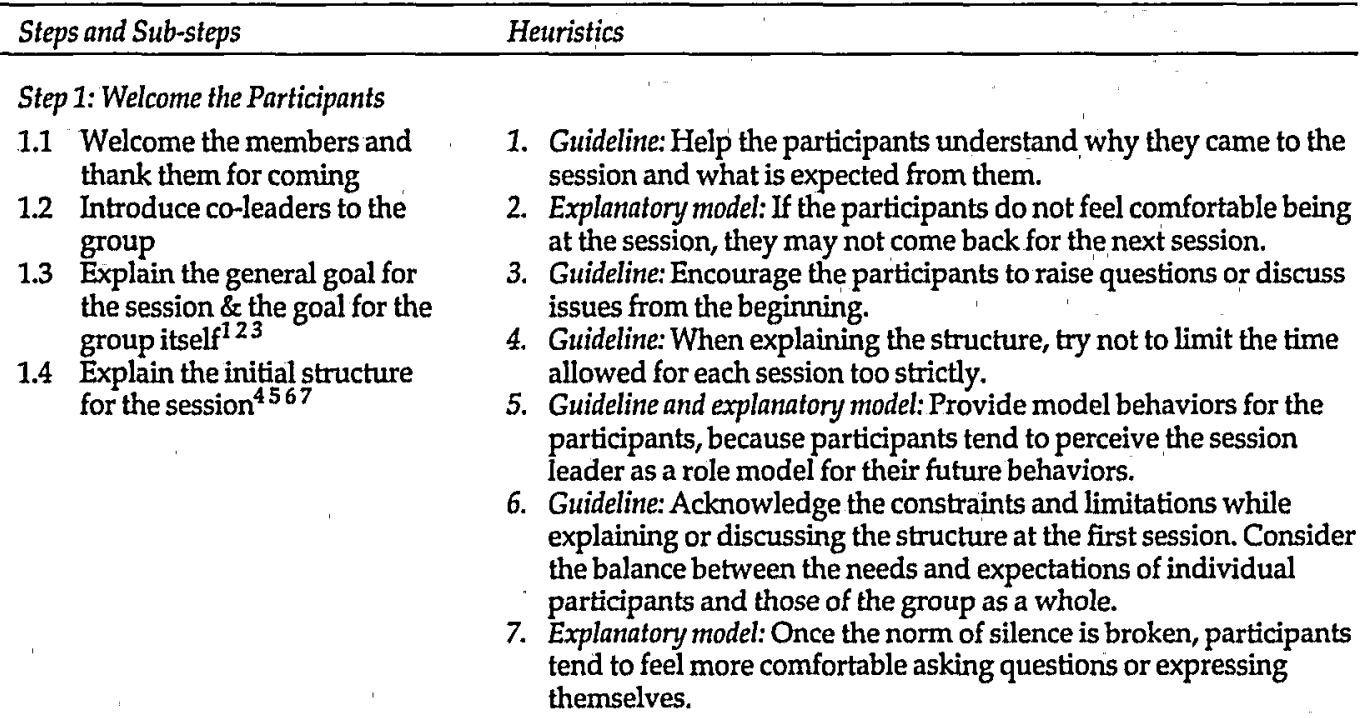

\section{Step 2: Get to Know One Another through Ice Breakers 8910}

2.1 Pair-off twos ${ }^{11} 12$

2.2 Let members in each pair interview each other ${ }^{1314}$

2.3 Let each member introduce his or her partner to the group

2.4 Repeat every member's name to make sure the group has everybody's name ${ }^{1516}$
8. Metacognitive rule: Deciding which icebreaker activity to use is rather a matter of preferences. Since this step is instrumental, the details of the activity do not matter as long as the overall activity serves as an icebreaker at this early stage of group formation.

9. Guideline: Participate in the ice breakers yourself with the rest of the group.

10. Explanatory model: By seeing the session leader take part in the group exercise just like everyone else, participants tend to accept him or her as part of the group and thus feel more comfortable following his or her lead.

11. Explanatory model: Compared to other types of ice breakers where people take turns to introduce themselves, participants tend to pay more attention to one another and be more active speakers.

12. Explanatory model: When the participants know that they have to introduce someone to the rest of the group, they tend to be more willing to get to know that person.

13. Guideline: Ask the participants to talk about at least one thing that they normally would not tell others.

14. Explanatory model: This exercise helps the participants get used to talking about things that they might not otherwise, which is exactly what they will have to do throughout the sessions.

15. Guideline: Try to remember the participants' names and call each member by his or her first name as often as possible.

16. Explanatory model: Using participants' names frequently communicates that you respect and care about them.

17. Descriptive model: Interpersonal theories.

\section{Step 3: Set Group Norms}

3.1 Explain the meaning and need for setting group norms

3.2 Provide examples of group norms (e.g., confidentiality)

3.3 Include all members in group discussion ${ }^{18} 1920$
18. Guideline: If one member wants to talk a lot, set up a limit (e.g., "we've been discussing it for a while. Let's talk it for 5 more minutes and then save it for the next time.").

19. Explanatory model: Guideline 18 allows the leader to establish a structure and assure the members that the leader is there to make sure that the group keeps running. 


\section{Appendix $C \square$ (continued)}

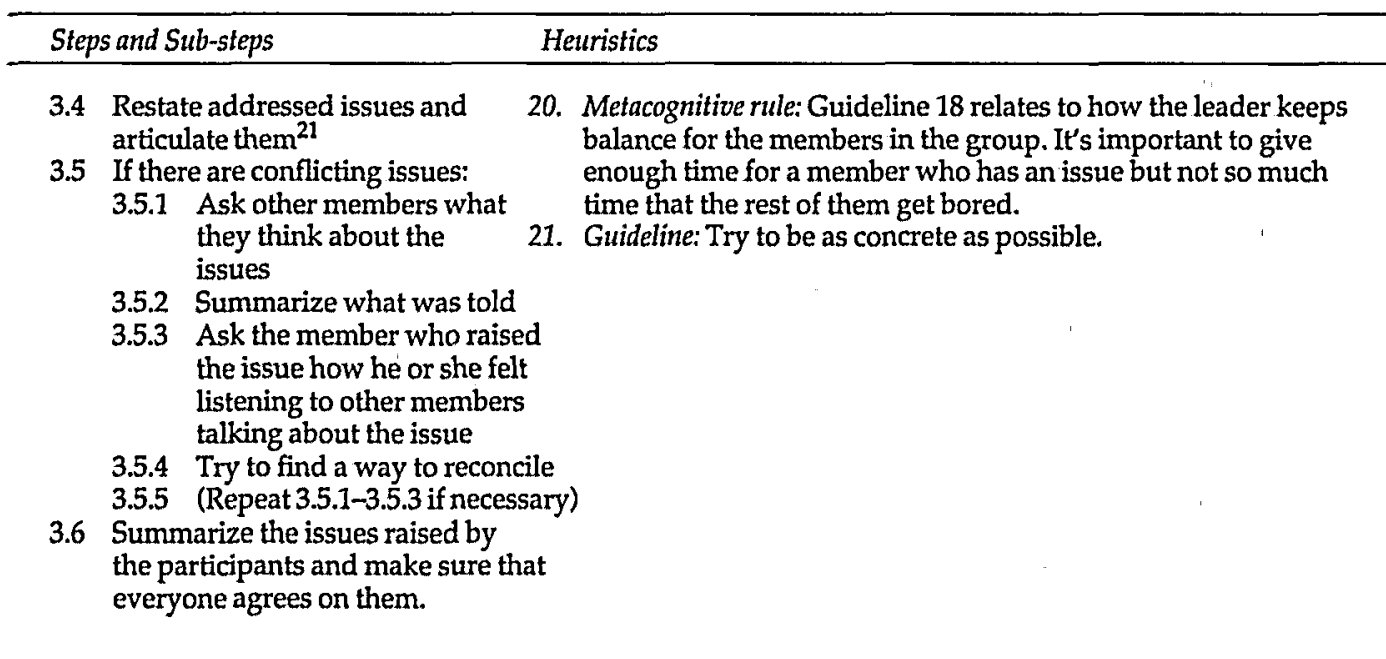

Step 4: Set Group Goals22

4.1 Explain the meaning of and needs for goal setting

4.2 Let each participant express his or her goals and expectations for the session 2324

4.3 Restate what each person says, and ask that it be articulated if necessary

4.4 Summarize the goals addressed by the entire group, and try to find commonalities among them.
22. Metacognitive rule: Developing group norms and setting group goals are closely interrelated steps. One cannot achieve one without carefully considering the other.

23. Guideline: Help the participants understand that the goals and expectations are only temporary and thus they can be revisited and changed over time.

24. Metacognitive rule: What is important is the process of setting the goals, rather than the goals themselves. Thus, the emphasis is on the quality of the process.
Step 5: Provide Feedback

5.1 Ask the participants about how they feel about the group 25262728

5.2 Restate what each person says and ask him or her to articulate it if necessary

5.3 Summarize each person's comments and provide feedback
25. Guideline: Try not to be too formal (e.g., taking turns in speaking), yet give mild pressures to the participants to engage in debriefing.

26. Guideline: For silent participants, try to understand the "quality" of their silence. It may be because of their personality, temporary feelings or moods, etc.

27. Guideline: Allow the participants to decide the initial pattern of interaction; respect their preferences to certain a degree.

28. Metacognitive Rule: One of the important roles of the session leader is to interact with the participants in a way that is consistent with what he or she expects from them, especially during the beginning steps.
Step 6: Close the Session

6.1 Summarize the session

6.2 Announce the main activities for the next session

6.3 Thank the members for their taking time for the session and show appreciation for their contribution 29
29. Guideline: Differentiate the degree of appreciation according to the quality of participation and the participant. (It is not appropriate to thank someone who has been always talkative for making a lot of comments during the session. However, it is important to acknowledge a participant for a comment if it was his or her first comment, and encourage further participation.) 


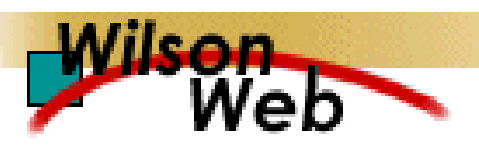

\section{COPYRIGHT INFORMATION}

TITLE: Formative Research on the Heuristic Task Analysis

Process

SOURCE: Educ Technol Res Dev 51 no4 2003

WN: 0300403447001

The magazine publisher is the copyright holder of this article and it is reproduced with permission. Further reproduction of this article in violation of the copyright is prohibited.

Copyright 1982-2004 The H.W. Wilson Company. All rights reserved. 